\title{
The radiation dose to overweighted patients undergoing myocardial perfusion SPECT can be significantly reduced: validation of a linear weight-adjusted activity administration protocol
}

\author{
Jenny Oddstig, PhD, ${ }^{a}$ Cecilia Hindorf, $\mathrm{PhD},{ }^{\mathrm{a}}$ Fredrik Hedeer, $\mathrm{MD},{ }^{\mathrm{b}}$ Jonas Jögi, \\ MD, PhD, ${ }^{b}$ Håkan Arheden, MD, PhD, ${ }^{b}$ Magnus J. Hansson, MD, PhD, ${ }^{b}$ and \\ Henrik Engblom, MD, PhD ${ }^{b}$ \\ a Department of Radiation Physics, Skåne University Hospital and Lund University, Lund, Sweden \\ b Department of Clinical Physiology and Nuclear Medicine, Skåne University Hospital and Lund \\ University, Lund, Sweden
}

Received Nov 18, 2015; accepted Jun 25, 2016

doi: 10.1007/s12350-016-0628-7

Background. Large body size can cause a higher proportion of emitted photons being attenuated within the patient. Therefore, clinical myocardial perfusion SPECT (MPS) protocols often include unproportionally higher radioisotope activity to obese patients. The aim was to evaluate if a linear weight-adjusted low-dose protocol can be applied to obese patients and thereby decrease radiation exposure.

Methods and result. Two hundred patients ( $>110 \mathrm{~kg}$, BMI 18-41, $[n=69], \leq 110 \mathrm{~kg}$, BMI 31-58, $[n=131]$ ) underwent ${ }^{99 m}$ Tc-tetrofosmin stress examination on a Cadmium Zinc Telluride or a conventional gamma camera using new generations of reconstruction algorithm (Resolution Recovery). Patients $<110 \mathrm{~kg}$ were administered $2.5 \mathrm{MBq} / \mathrm{kg}$, patients between 110 and $120 \mathrm{~kg}$ received $430 \mathrm{MBq}$ and patients $>120 \mathrm{~kg}$ received $570 \mathrm{MBq}$ according to clinical routine. Patients $>110 \mathrm{~kg}$ had $130 \%$ total number of counts in the images compared to patients $<110 \mathrm{~kg}$. Recalculating the counts to correspond to an administered activity of 2.5 MBq/kg resulted in similar number of counts across the groups. Image analyses in a subgroup with images corresponding to high activity and $2.5 \mathrm{MBq} / \mathrm{kg}$ showed no difference in image quality or ischemia quantification.

Conclusion. Linear low-dose weight-adjusted protocol of $2.5 \mathrm{MBq} / \mathrm{kg}$ in MPS can be applied over a large weight span without loss of counts or image quality, resulting in a significant reduction in radiation exposure to obese patients. ( $J$ Nucl Cardiol 2017;24:1912-21.)

Key Words: Myocardial perfusion imaging: SPECT $\cdot$ CZT detector $\cdot$ conventional gamma camera $\cdot$ image quality $\cdot$ radiation dose

\section{See related editorial, pp. 1922-1925}

Funding This work was supported in part by research funding from Region of Scania, Swedish Heart-Lung Foundation, and Lund University Faculty of Medicine.

Reprint requests: Henrik Engblom, MD, PhD, Department of Clinical Physiology and Nuclear Medicine, Skåne University Hospital and Lund University, 221 85, Lund, Sweden; henrik.engblom@med.lu.se $1071-3581 / \$ 34.00$

Copyright $\odot 2016$ The Author(s). This article is published with open access at Springerlink.com

1912

\section{INTRODUCTION}

Ischemic heart disease (IHD) is one of the most common causes of death in the western world ${ }^{1}$ and a major cause of heart failure. ${ }^{2}$ Presence of stress-induced myocardial ischemia due to significant coronary stenosis has been shown to be of fundamental clinical importance for patient $\operatorname{prognosis}^{3,4}$ and need for revascularization therapy. ${ }^{5}$ Current ESC/EACTS (European Society of Cardiology/European Association for Cardio-thoracic Surgery) guidelines ${ }^{6}$ therefore strongly recommend having a stress examination performed in patients with suspected stable coronary artery disease 
(CAD) before decision for revascularization therapy is taken.

Myocardial perfusion single-photon emission computed tomography (MPS) is a well-established noninvasive imaging technique for detection of myocardial ischemia. Recently, a new generation of gamma camera systems was introduced based on a novel detector technology utilizing semiconductor detectors of Cadmium Zinc Telluride (CZT) ${ }^{7,8}$ Furthermore, a new generation of reconstruction methods for the conventional gamma cameras was recently introduced referred to as Ordered Subset Expectation Maximization with depth-dependent Resolution Recovery (OSEMRR). ${ }^{9}$ Both CZT detectors and OSEM-RR can be used to reduce the radiation exposure to nonobese patients $^{10-12}$ in accordance with ALARA (As Low As Reasonably Achievable). ${ }^{13}$

A large body size may cause a higher proportion of emitted photons being attenuated and scattered within the patient resulting in reduced number of primary photons reaching the detector. In order to compensate for this, clinical protocols often include administration of proportionally higher radioisotope activity to patients with higher body weight in accordance with American Society of Nuclear Cardiology (ASNC) guidelines, ${ }^{14}$ whereas others recommend a fixed dose to all patients regardless of weight. ${ }^{15}$ Both strategies result in a large variation in absorbed dose with potentially unnecessary high radiation exposure to patients with overweight/ obesity and low body weight, respectively. Body mass index (BMI) is an index of weight-for-height that is commonly used to classify overweight and obesity in adults. The World Health Organization (WHO) definition is as follows: a BMI $\geq 25$ is overweight and a $\mathrm{BMI} \geq 30$ is obesity.

We have previously demonstrated that the new CZT technology enables a radiation dose reduction in nonobese patients by implementing a low-dose protocol, $2.5 \mathrm{MBq} / \mathrm{kg}$ body weight. ${ }^{12}$ Other studies have also demonstrated the possibility of low-dose protocol using new CZT technology or new generation of reconstruction algorithms OSEM-RR on conventional gamma cameras in nonobese patients. ${ }^{16-20}$ A recent study indicates the possibility of dose reduction also for obese patients with a fixed activity using the CZT technology. ${ }^{21}$

The aim of the present study was to evaluate whether a similar linear administration schedule can be extended to all adult patients, not only nonobese, on both CZT cameras and conventional gamma cameras using resolution recovery reconstruction, in order to enable a decrease in radiation exposure to obese patients.

\section{MATERIALS AND METHODS}

\section{Study Population and Study Design}

The study included 200 patients referred for MPS due to known or suspected coronary artery disease. All patients underwent a ${ }^{99 \mathrm{~m}} \mathrm{Tc}$-tetrofosmin stress examination, according to clinical routine. The stress was accomplished either by a bicycle ergometer test or pharmacologic stress. The amount of radioisotope was administered according to body weight for patients below $110 \mathrm{~kg}(2.5 \mathrm{MBq} / \mathrm{kg})$. According to clinical routine, a fixed activity of 430 and $570 \mathrm{MBq} \pm 10 \%$ was used for patients between 110 and $120 \mathrm{~kg}$ and above $120 \mathrm{~kg}$, respectively. The syringe was measured before and after injection of ${ }^{99 \mathrm{~m}} \mathrm{Tc}$-tetrofosmin to calculate the specific administered activity. A subset of the included patients with a body weight over $110 \mathrm{~kg}$ were also scanned with a reduced acquisition time corresponding to an injected activity of 2.5 MBq/kg of body weight.

Rest examination was performed if the stress examination images were interpreted as abnormal. The rest examination was undertaken according to a predefined, in-house developed time schedule designed to meet the ASNC recommended guidelines of 3 times more activity in the rest examination in a 1-day protocol for patients $<120 \mathrm{~kg}$. Thus, individually adjusted activity was administered at rest depending on the duration between the two examinations, with a minimum of 2 hours, resulting in decreased activity with increased duration. Patients $>120 \mathrm{~kg}$ was examined with a two-day protocol since the total activity otherwise would exceed what the Swedish regulations allow for MPS.

The number of counts in the left ventricle was analyzed on all images, and on a subset of images the perceived quality as well as a quantitative diagnostic analysis was performed. All patients provided written informed consent to participate in the study and the study was approved by the regional ethics committee.

\section{Image Acquisition and Reconstruction}

The scan was performed approximately 1 hour after the injection with either a CZT gamma camera (Discovery NM 530c, GE Healthcare) or a conventional gamma camera (Ventri, GE Healthcare) dedicated to cardiac imaging. Patients were scanned in supine position with the arms positioned over the head. Patients with high uptake of ${ }^{99 \mathrm{~m}} \mathrm{Tc}$-tetrofosmin in the abdomen were rescanned after intake of water and a walk to avoid activity in the abdomen in the images. ECG-gated images were acquired using 8 bins for both cameras. No correction for scatter or attenuation was performed. The images were reconstructed on a dedicated workstation (Xeleris, GE Healthcare). All images were reconstructed and reformatted into the standard axes (short axis, vertical long axis, horizontal long axis), and polar maps of the left ventricle were created.

CZT camera. Acquisition on the CZT camera after stress was made with a scan time of 475 seconds based on the study by Herzog et al. ${ }^{22}$ A $15 \%$ symmetrical energy window at 
$140 \mathrm{keV}$ was used. The images were reconstructed with an iterative reconstruction algorithm. For gated stress images, Maximum Likelihood Estimation Method (MLEM) 30 iterations and Green OSL regularization alpha 0.4 and beta 0.4 were used and the images were postfiltered with a Butterworth filter with cut-off frequency of 0.4 and power 10 . For the nongated stress images, MLEM 40 iterations and Green OSL regularization alpha parameter of 0.51 and a beta of 0.3 were used and the images were postfiltered with a Butterworth filter with a cut-off frequency of 0.37 and a power of 7 . All reconstruction parameters used followed the recommendations from the manufacturer. The images on the CZT camera were acquired in list mode, which enables the images to be resampled into a shorter acquisition via the list mode tool Lister (Xeleris, GE Healthcare). Images for a subgroup of eight patients with a body weight over $110 \mathrm{~kg}$ (the patients administered with fixed activities) were resampled into an acquisition time that would correspond to an administered activity of $2.5 \mathrm{MBq} / \mathrm{kg}$.

Conventional gamma camera. Acquisition on the conventional gamma camera after stress was performed with the detectors in L-mode using a $64 \times 64$ matrix and a $20 \%$ symmetrical energy window over the $140 \mathrm{keV}$ photon peak. Sixty (60) projections were acquired in a total angular range of 180 degrees with a stop condition of 25 seconds per projection. The images acquired on the conventional gamma camera were reconstructed with a resolution recovery OSEM algorithm (Evolution, GE Healthcare) with 12 iterations and 10 subsets and postfiltered with a Butterworth filter with a cut-off frequency of 0.4 and a power of 10 in stress. A second acquisition with a shorter time per angle to correspond to an administered activity of $2.5 \mathrm{MBq} / \mathrm{kg}$ body weight was performed on a subgroup of eight patients with a body weight over $110 \mathrm{~kg}$.

\section{Image Analysis}

The total number of counts in the left ventricle was derived for all studies by delineating the ventricle by automatic segmentation. The number of counts in the acquired images, $C_{a c q}$, for both cameras was recalculated to correspond to the number of counts that would have been acquired if the administered activity would have been $2.5 \mathrm{MBq} / \mathrm{kg}, C_{2.5 \mathrm{MBq} / \mathrm{kg}}$ (Eq. 1). $m_{\text {patient }}$ is the patient's body weight and $A_{0}$ is the administered activity to the patient.

$$
C_{2.5 \mathrm{MBq} / \mathrm{kg}}=C_{\text {acquired }} \cdot \frac{2.5 \cdot m_{\text {patient }}}{A_{0}} .
$$

For a subgroup of 16 patients ( 8 examined on CZT and 8 examined on conventional gamma camera), the image quality (qualitative and quantitative) for the images of the current administration schedule and for images corresponding to an administered activity of $2.5 \mathrm{MBq} / \mathrm{kg}$ was evaluated. Two experienced observers reviewed the images in a randomized order. The readers were blinded to each other and all patient data as well as to if the data set were current administration schedule with fixed activities or $2.5 \mathrm{MBq} / \mathrm{kg}$. The image quality was visually graded according to a four-point scale
( 1 = poor, 2 = adequate, $3=$ good, $4=$ excellent $)$. The following variables were considered when grading the image quality: noise level, the homogeneity of uptake as seen on both the tomographic short- and long-axis slices and on the polar plot representation. For quantification of ischemia, summed stress score was manually determined. The analysis was performed based on the 17-segment model of the left ventri$\mathrm{cle}^{23}$ using the myocardial perfusion SPECT module in the software Segment as previously described. ${ }^{24}$

The total number of counts in the left ventricle was derived also for the rest images. The counts from the stress examination were decay-corrected and subtracted from the rest image. In the same way as for the stress image, the counts from the rest images were recalculated to correspond to the number of counts that would have been acquired if the administered activity would have been $3.1 \mathrm{MBq} / \mathrm{kg}$, (Eq. 1). $3.1 \mathrm{MBq} / \mathrm{kg}$ is clinically used for rest examination since the uptake in the ventricle is lower in rest than in stress. For the patients examined with a two-day protocol was the number of counts recalculated to correspond to an injected activity of $3.1 \mathrm{MBq} /$ $\mathrm{kg}$.

\section{Dosimetry}

The mean absorbed dose to whole body $(\bar{D})$ as a function of body weight was calculated for our current clinical administration schedule, administered activity according to body weight $(2.5 \mathrm{MBq} / \mathrm{kg})$, the activity recommended by the ASNC (444 MBq for patients below $70 \mathrm{~kg}, 444 \mathrm{MBq}+$ 11.5 MBq/kg body weight for patients over $70 \mathrm{~kg})^{25}$ and by the European Association of Nuclear Medicine (EANM) $(500 \mathrm{MBq})^{15}$ (Eq. 2). The residence time ( $\tau$, the number of decays in whole body per administered activity) for ${ }^{99 \mathrm{~m}} \mathrm{Tc}-$ tetrofosmin was taken from the International Commission on Radiological Protection (ICRP) publication $106 .{ }^{26}$ The $\mathrm{S}$ value (absorbed dose to whole body per decay in the whole body) was scaled according to body weight $\left(m_{\text {patient }}\right)$. The administered activity to the patient is denoted $A_{0}$. The mass of the phantom for which the original $\mathrm{S}$ value was determined ( $\left.m_{\text {phantom }}\right)$ equals to $73 \mathrm{~kg}$.

$$
D=A_{0} \cdot \tau \cdot S \cdot \frac{m_{\text {phantom }}}{m_{\text {patient }}} .
$$

\section{Statistical Analysis}

The data are presented as mean $\pm \mathrm{SD}$. To test for differences in number of counts between 4 and $2.5 \mathrm{MBq} / \mathrm{kg}$, a paired $t$ test was used. To test for differences with regards to image quality and summed stress score, the Wilcoxon signedrank test was used. Statistical significance was defined as $p<0.05$.

\section{RESULTS}

All 200 patients successfully underwent a stress examination, 82 by exercise and 118 by pharmacologic. Thirty-three (33) of the patients had a body weight over $120 \mathrm{~kg}$ (BMI 31-58), 36 patients had a body weight 
Table 1. The table shows the number of patients in the three different weight groups and on which camera their images were acquired [body mass index (BMI)]. In total were 200 patients included in this study

\begin{tabular}{lcccc}
\hline & \multicolumn{1}{c}{$<\mathbf{1 0 ~ k g}$} & $\mathbf{1 1 0 - 1 2 0 ~ k g}$ & $>120 \mathbf{~ k g}$ & \multicolumn{1}{c}{ Total } \\
\hline $\begin{array}{l}\text { Conventional } \\
\text { gamma camera } \\
\text { (BMI) }\end{array}$ & $50(18-41$, mean 29) & $19(32-42$, mean 37) & $29(31-58$, mean 42$)$ & $98(18-58$, mean 34) \\
$\begin{array}{l}\text { CZT camera (BMI) } \\
\text { Total BMI }\end{array}$ & $81(21-36$, mean 29) & $17(31-43$, mean 36) & $4(31-37$, mean 34) & $102(21-36$, mean 30) \\
& $131(18-41$, mean 29) & $36(31-43$, mean 36) & $33(31-58$, mean 41) & $200(18-58$, mean 32)
\end{tabular}

Table 2. Baseline characteristics of all included patients ( $N$ or mean $\pm S D)$

\begin{tabular}{lcc}
\hline & Exercise stress $(\boldsymbol{n}=\mathbf{8 2})$ & Pharmacological stress $(\boldsymbol{n}=\mathbf{1 1 8})$ \\
\hline Age $($ years $)$ & $64 \pm 10$ & $66 \pm 10$ \\
Male & $56(68 \%)$ & $87(74 \%)$ \\
Female & $26(32 \%)$ & $31(26 \%)$ \\
BMI $\left(\mathrm{kg} / \mathrm{m}^{2}\right)$ & $31 \pm 7(21-52)$ & $33 \pm 7(18-58)$ \\
Body weight $(\mathrm{kg})$ & $94 \pm 22(55-156)$ & $102 \pm 23(51-193)$ \\
Diabetes & $16(20 \%)$ & $42(36 \%)$ \\
Hyperlipidemia & $38(46 \%)$ & $60(51 \%)$ \\
Hypertension & $46(56 \%)$ & $79(67 \%)$ \\
Smoking & $55(67 \%)$ & $88(75 \%)$ \\
Family history of CAD & $28(34 \%)$ & $45(38 \%)$ \\
History of MI & $21(26 \%)$ & $35(30 \%)$ \\
PCI & $21(26 \%)$ & $37(31 \%)$ \\
CABG & $9(2 \%)$ & $23(19 \%)$ \\
\hline
\end{tabular}

between 110 and $120 \mathrm{~kg}$ (BMI 32-42), and 131 patients had a body weight below $110 \mathrm{~kg}$ (BMI 18-41). The injected activities were (mean $\pm \mathrm{SD}) 2.57 \pm 0.21 \mathrm{MBq} /$ $\mathrm{kg}, 413 \pm 29 \mathrm{MBq}$ and $583 \pm 53 \mathrm{MBq}$ at stress for the three weight protocols $<110,110-120$, and $>120 \mathrm{~kg}$, respectively. Ninety-eight (98) of the patients (50 patients with a body weight $<110 \mathrm{~kg}$ (BMI mean 29), 19 between 110 and $120 \mathrm{~kg}$ (BMI mean 37), and 29 with a weight $>120 \mathrm{~kg}(\mathrm{BMI}$ mean 42) were examined on the conventional gamma camera and 102 patients (81 patients with a body weight $<110 \mathrm{~kg}$ (BMI mean 29), 17 between 110 and $120 \mathrm{~kg}$ (BMI mean 36), and 4 with a weight $>120 \mathrm{~kg}$ (BMI mean 34) on the CZT camera (Table 1). Patient characteristics are shown in Table 2.

\section{NUMBER OF COUNTS}

The total numbers of reconstructed counts in the left ventricle for the stress examination on the CZT camera were $\quad 1.63 \mathrm{E} 5 \pm 0.37 \mathrm{E} 5, \quad 2.21 \mathrm{E} 5 \pm 0.76 \mathrm{E} 5, \quad$ and $2.99 \mathrm{E} 5 \pm 0.61 \mathrm{E} 5$ for patients $<110 \mathrm{~kg}, 110-120 \mathrm{~kg}$, and $>120 \mathrm{~kg}, \quad$ respectively $\quad$ (Figure $1 \mathrm{~A}$ ). The recalculation of the total number of counts to correspond to an injected activity of $2.5 \mathrm{MBq} / \mathrm{kg}$ for two patient groups $>110 \mathrm{~kg}$ administered with fixed activities was $1.58 \mathrm{E} 5 \pm 0.43 \mathrm{E} 5$ for the CZT camera, which did not differ significantly from the number of counts in patients $<110 \mathrm{~kg}(p=0.63$; Figure 1B). Recalculating the counts in the left ventricle for the rest examination to correspond to an injected activity of $3.1 \mathrm{MBq} / \mathrm{kg}$ showed no statistical difference between patients $>$ $110 \mathrm{~kg}$ and patients $<110 \mathrm{~kg}(\mathrm{p}=0.59$, Figure $1 \mathrm{C})$.

The total numbers of reconstructed counts for the stress examination on the conventional gamma camera were 4.29E5 $\pm 1.48 \mathrm{E} 5,5.94 \pm 1.40 \mathrm{E} 5$, and $8.26 \pm 2.96$ E5 for patients $<110,110-120$, and $>120 \mathrm{~kg}$, respectively (Figure 2A). Recalculating the total number of counts corresponding to an administered activity of $2.5 \mathrm{MBq} / \mathrm{kg}$ for the two patient groups $>110 \mathrm{~kg}$ resulted in a number of counts of $4.42 \mathrm{E} 5 \pm 1.43 \mathrm{E} 5$ for the conventional gamma camera, which did not differ from the number of counts in patients $<110 \mathrm{~kg}$ ( $p=0.24)$ (Figure 2B). The recalculation of counts in the left ventricle for the rest examination to correspond to an 


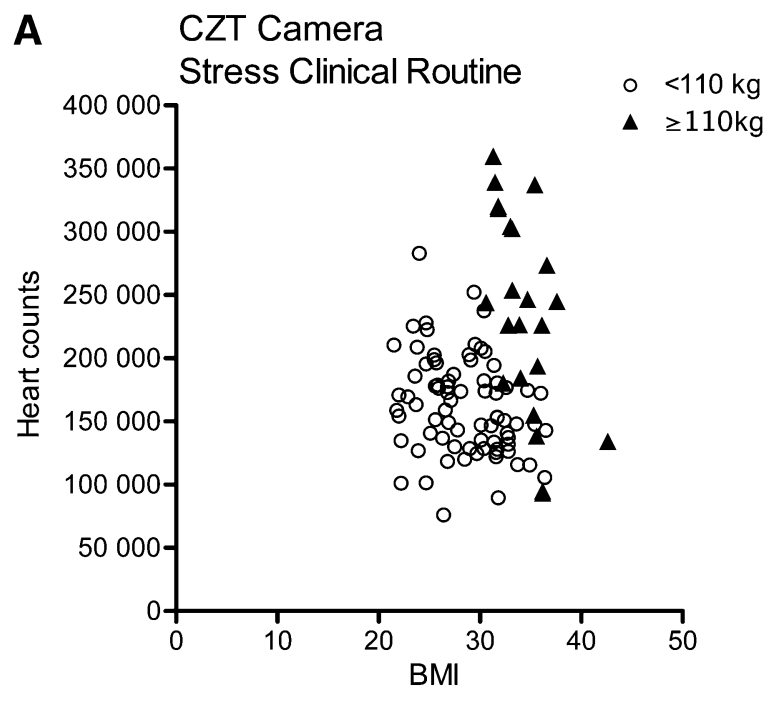

B Stress Recalculated to $2.5 \mathrm{Mbq} / \mathrm{kg}$

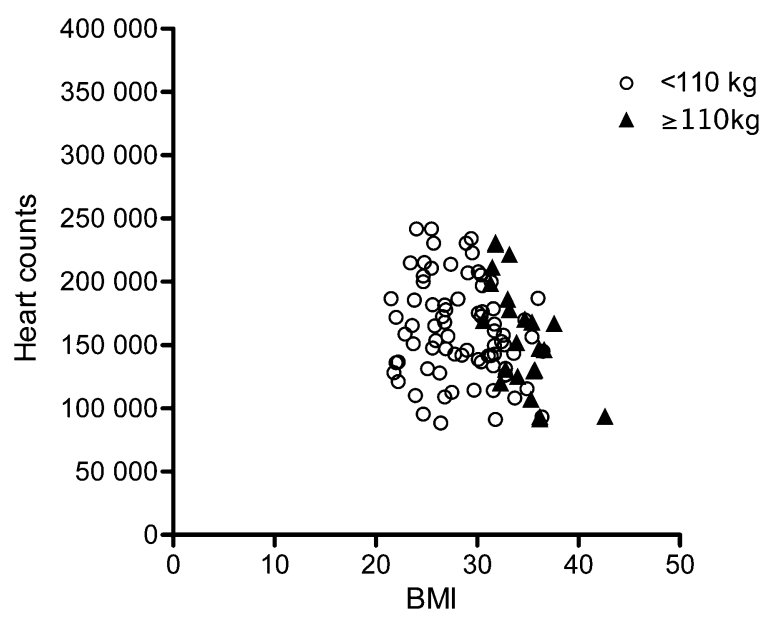

C Rest Recalculated to $3.1 \mathrm{Mbq} / \mathrm{kg}$

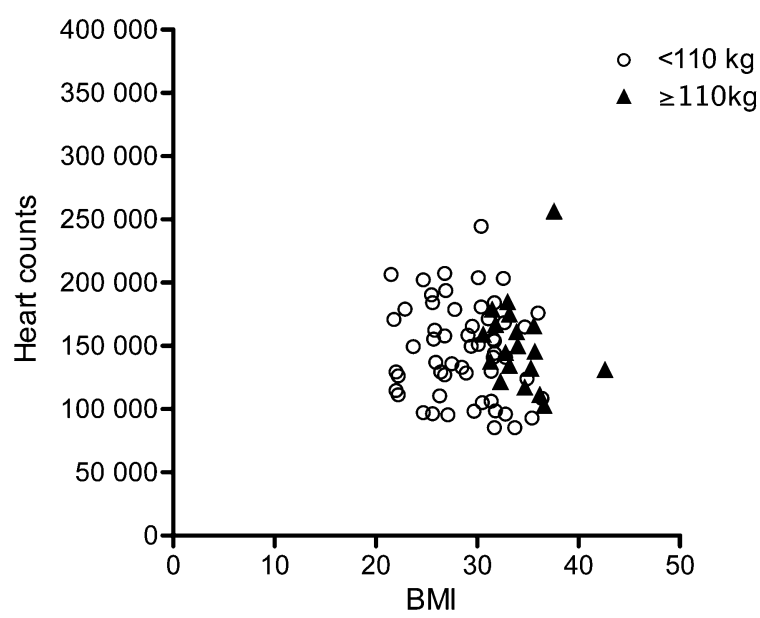

4Figure 1. The total number of reconstructed counts in the left ventricle on the CZT camera for the A current activity administration schedule at stress $(<110 \mathrm{~kg}: 2.5 \mathrm{MBq} / \mathrm{kg}, 110-$ $120 \mathrm{~kg}$ : $430 \mathrm{MBq},>120 \mathrm{~kg}$ : $570 \mathrm{MBq})$, B if $2.5 \mathrm{MBq} / \mathrm{kg}$ would have been administered at stress, and $\mathbf{C}$ if $3.1 \mathrm{MBq} / \mathrm{kg}$ would have been administrated at rest.

injected activity of $3.1 \mathrm{MBq} / \mathrm{kg}$ showed no statistical difference between patients $>110 \mathrm{~kg}$ and patients $<110 \mathrm{~kg}$ ( $p=0.14$; Figure 2C).

The acquisition of the stress examination was undertaken at a mean of 56 minutes after the injection for exercise and 67 minutes after the injection for pharmacological stress. For the conventional camera, the counts in the myocardium were 5.29E5 $\pm 2.63 \mathrm{E} 5$ and $4.50 \mathrm{E} 5 \pm 2.65 \mathrm{E} 5$ for exercise and pharmacological stress, respectively ( $p$ value $=0.16$ ). For the CZT camera, the counts in the myocardium were $1.90 \mathrm{E} 5 \pm 5.96 \mathrm{E} 4$ and 1.69E5 \pm 5.64E 4 for exercise and pharmacological stress, respectively ( $p$ value $=0.07$ )

\section{IMAGE QUALITY AND ISCHEMIA SCORE}

Sixteen of the patients with a body weight over $110 \mathrm{~kg}$ (112-193 kg, BMI 31-58) were reimaged in the conventional gamma camera, or alternatively, and the listmode files from the CZT camera were resampled to create images that correspond to an injected activity of $2.5 \mathrm{MBq} / \mathrm{kg}$. The scores of the image quality for the two reviewers are shown in Table 3. The mean value of the evaluated image quality for the two administration schedules was equal $(p=1.0)$. Examples of the image quality for the current administration protocol and a liner protocol of $2.5 \mathrm{MBq} / \mathrm{kg}$ can be seen in Figure 3 .

There was no significant difference in SSS (summed stress score) $(p=0.27)$ for quantification of ischemia when comparing images reconstructed to reflect $4 \mathrm{MBq} /$ $\mathrm{kg}$ (median 2.0, range 0-36) with images reconstructed to reflect $2.5 \mathrm{MBq} / \mathrm{kg}$ (median 3.5, range 0-35; bias $\pm \mathrm{SD}=1.1 \pm 3.5$ ). The interobserver variability of SSS was $0.7 \pm 1.8$.

\section{Radiation Exposure}

Figure 4 shows the mean absorbed dose to whole body as a function of body weight when administered according to the ASNC guidelines, the EANM guidelines, our current activity administration schedule, and for an administration of $2.5 \mathrm{MBq} / \mathrm{kg}$. 


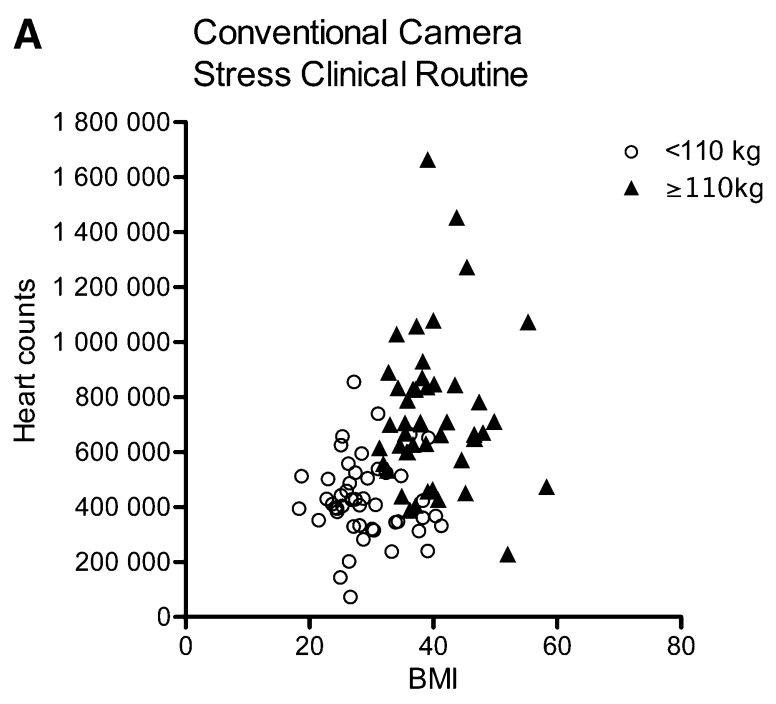

B Stress Recalculated to $2.5 \mathrm{Mbq} / \mathrm{kg}$
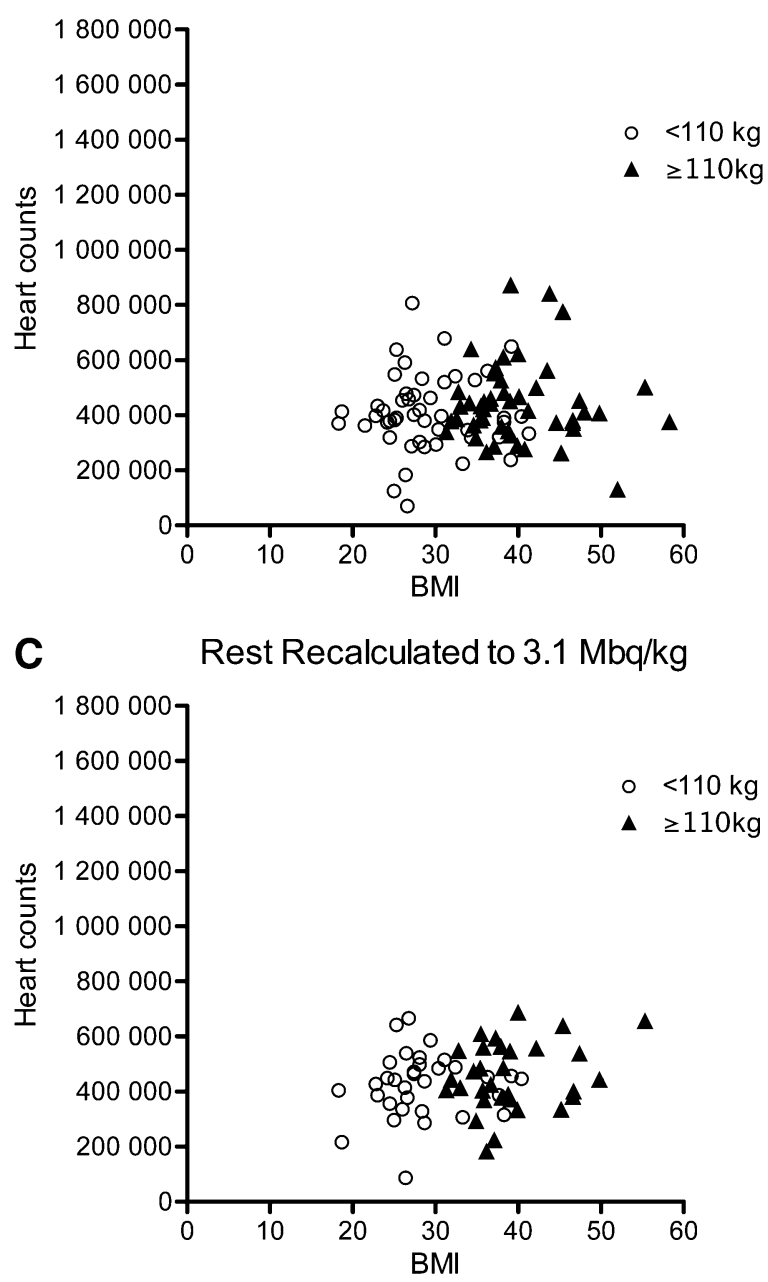

4Figure 2. The total number of counts in the left ventricle for the conventional gamma camera for the $\mathbf{A}$ current activity administration schedule at stress $(<110 \mathrm{~kg}: 2.5 \mathrm{MBq} / \mathrm{kg}, 110-$ $120 \mathrm{~kg}$ : $430 \mathrm{MBq},>120 \mathrm{~kg}$ : $570 \mathrm{MBq})$, B if $2.5 \mathrm{MBq} / \mathrm{kg}$ would have been administered at stress, and $\mathbf{C}$ if $3.1 \mathrm{MBq} / \mathrm{kg}$ would have been administrated at rest.

The effective dose for the stress examination using a linear administration schedule of $2.5 \mathrm{MBq} / \mathrm{kg}$ body weight for a $120 \mathrm{~kg}$ patient would decrease from $4.0 \mathrm{mSv}(570 \mathrm{MBq})$ to $2.1 \mathrm{mSv}(300 \mathrm{MBq})$ in stress.

\section{DISCUSSION}

This study shows that it is possible to use a linear administration schedule of $2.5 \mathrm{MBq} / \mathrm{kg}$ body weight for MPS on patients with a body weight ranging from $51 \mathrm{~kg}$ to $193 \mathrm{~kg}$ (BMI 18-58) using a CZT gamma camera or a conventional gamma camera with a resolution recovery reconstruction algorithm. This will significantly reduce the effective dose to the patient compared to the EANM/ ASNC guidelines.

The findings in the present study show that the traditional proportionally higher administered activity or prolonged acquisition time for obese patients is not necessary for MPS. The higher proportion of attenuation and scattered photons in fat tissue had no detectable influence on image quality and ischemia quantification. Therefore, an administration schedule based on a linear increase of activity, $2.5 \mathrm{MBq} / \mathrm{kg}$, can be used also for obese patients without loss of counts due to attenuation. Using an administration schedule of $2.5 \mathrm{MBq} / \mathrm{kg}$ reduces the radiation dose with $30 \%$ in stress compared to the clinically used protocol for a $120 \mathrm{~kg}$ patient. Reducing the administered activity is not only beneficial for the patient but also advantageous for the staff taking care of the patient.

Millions of MPS studies are performed each year worldwide. Thus, reducing the administered activity will result in a decreased collective dose, both for patients and staff, and consequently a decreased risk associated with ionizing radiation exposure. Reducing the administered activity may also be important to prolong the use of MPS in the predicted worldwide deficiency of ${ }^{99 \mathrm{~m}} \mathrm{Tc}$.

Examining obese patients with the GE Discovery 530 CZT camera has been reported to be challenging $^{21,27}$ due to the limited quality field of view (QFOV) associated with this camera. For patients with a large body habitus, it may be difficult to obtain an 
Table 3. The result of the image quality for the current administration schedule with fixed activity (110-120 kg: $430 \mathrm{MBq},>120 \mathrm{~kg}: 570 \mathrm{MBq}$ ) and for images corresponding to an administered activity of $2.5 \mathrm{MBq} / \mathrm{kg}$ in 16 patients $(112-193 \mathrm{~kg}$, BMI $31-58)$. The first eight patients were examined on a convention gamma camera, BMI 34-58, and the last eight on a CZT camera, BMI 31-38

\begin{tabular}{|c|c|c|c|c|}
\hline \multirow[b]{2}{*}{ Patient } & \multicolumn{2}{|c|}{ Reviewer 1} & \multicolumn{2}{|c|}{ Reviewer $\mathbf{2}$} \\
\hline & $\begin{array}{l}\text { Current adm. } \\
\text { schedule }\end{array}$ & $2.5 \mathrm{MBq} / \mathrm{kg}$ & $\begin{array}{l}\text { Current adm. } \\
\text { schedule }\end{array}$ & $2.5 \mathrm{MBq} / \mathrm{kg}$ \\
\hline 1 & 3 & 3 & 3 & 2 \\
\hline 2 & 3 & 4 & 2 & 3 \\
\hline 3 & 3 & 4 & 3 & 3 \\
\hline 4 & 4 & 3 & 3 & 2 \\
\hline 5 & 3 & 4 & 2 & 3 \\
\hline 6 & 2 & 2 & 2 & 1 \\
\hline 7 & 4 & 3 & 3 & 3 \\
\hline 8 & 3 & 3 & 2 & 2 \\
\hline 9 & 3 & 3 & 3 & 3 \\
\hline 10 & 3 & 3 & 2 & 3 \\
\hline 11 & 4 & 4 & 4 & 4 \\
\hline 12 & 4 & 3 & 4 & 3 \\
\hline 13 & 3 & 3 & 3 & 3 \\
\hline 14 & 4 & 3 & 3 & 3 \\
\hline 15 & 3 & 4 & 3 & 4 \\
\hline 16 & 4 & 4 & 4 & 4 \\
\hline Mean: & 3.3 & 3.3 & 2.9 & 2.9 \\
\hline
\end{tabular}

acceptable position of the myocardium within the QFOV, where an off center position may introduce artifacts in the images. ${ }^{28}$ Therefore, it is important to show that a linear administration schedule of $2.5 \mathrm{MBq} /$ $\mathrm{kg}$ in obese patients can be used also for conventional MPS as has been shown in the present study. This is also valid for patients with large breasts that have to be examined with conventional MPS cameras for the same reason as above. ${ }^{29}$ It is difficult to triage if it will be possible to correctly position the patient by body weight or BMI but an experienced technologist can by visual inspection of the patient determine if a correct position will be possible as long as the heart is normally positioned in the body.

Using a linear schedule for administered activity in the stress examination will according to this protocol give an administered activity in the rest examination that is also linear to the body weight. For a 2-day protocol, the result from the stress examination found in this study could potentially also be used for the rest examination with a linear weight-based schedule.

\section{LIMITATIONS}

The findings in the present study should be interpreted in the light of limitations. Only a few patients $(n=4)$ with a weight $>120 \mathrm{~kg}$ were examined on the CZT camera (17 patients with a body weight between 110 and 120 were included). This was due to the fact that most of these patients could not be examined in this camera because the heart could not be positioned within the fixed QFOV related to the body habitus of these patients.

\section{NEW KNOWLEDGE GAINED}

Linear low-dose administration of ${ }^{99 \mathrm{~m}}$ Tc-tetrofosmin for MPS is feasible over a wide range of body weights including obese patients for examination not only with new generation of gamma cameras, CZT, but also using a conventional gamma camera applying the new generation of reconstruction algorithms taking into account depth-dependent OSEM-Resolution Recovery. 


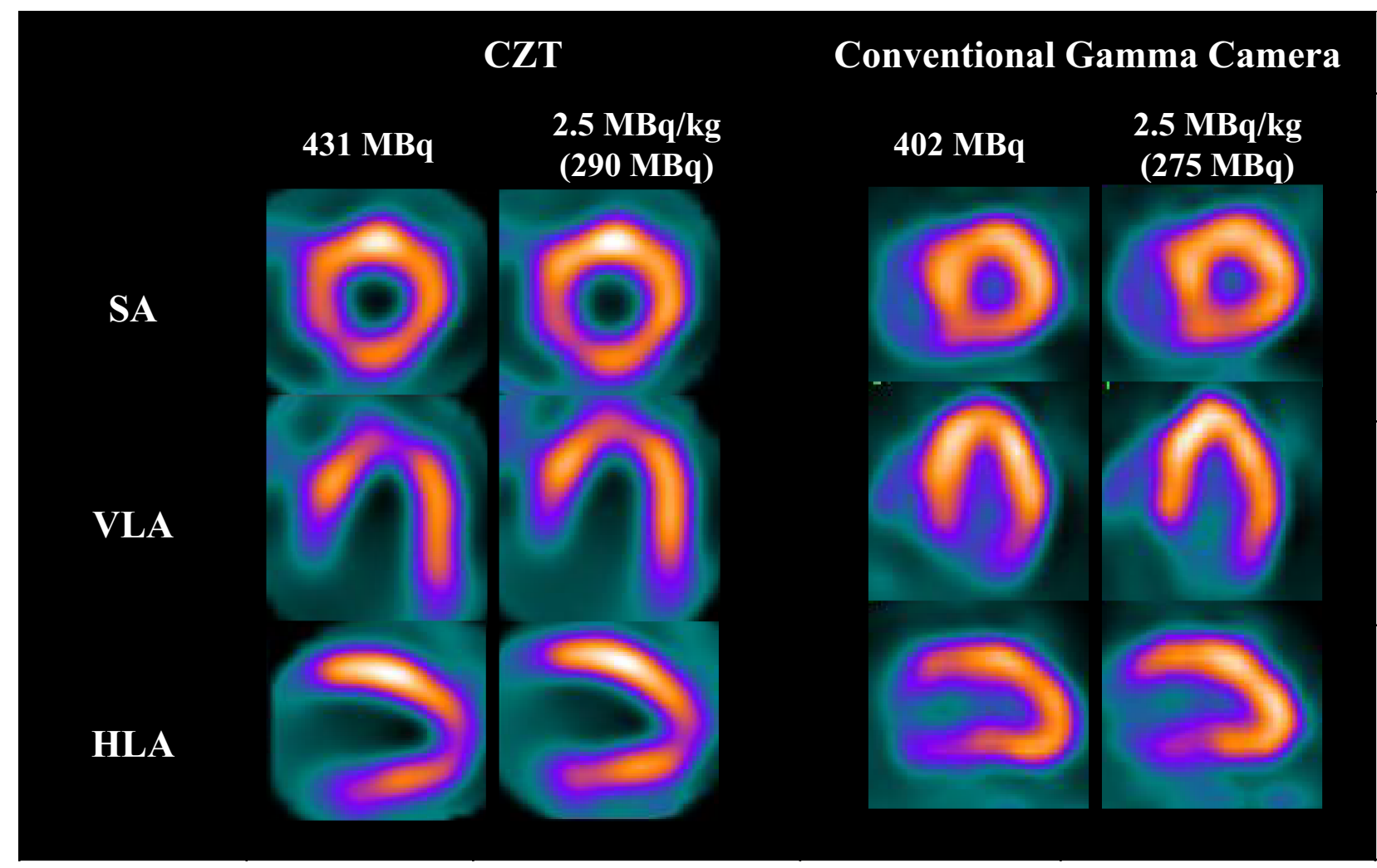

Figure 3. The image quality (stress examination) for two patients administered with $431 \mathrm{MBq}$ (scanned on the CZT camera) and $433 \mathrm{MBq}$ (scanned on the conventional gamma camera), according to the current protocol. The scanning was performed to current protocol ( 475 seconds for the CZT and 25 seconds/projection on the convention gamma camera) and with a reduced scan time to correspond to an administered activity of $2.5 \mathrm{MBq} / \mathrm{kg}$.

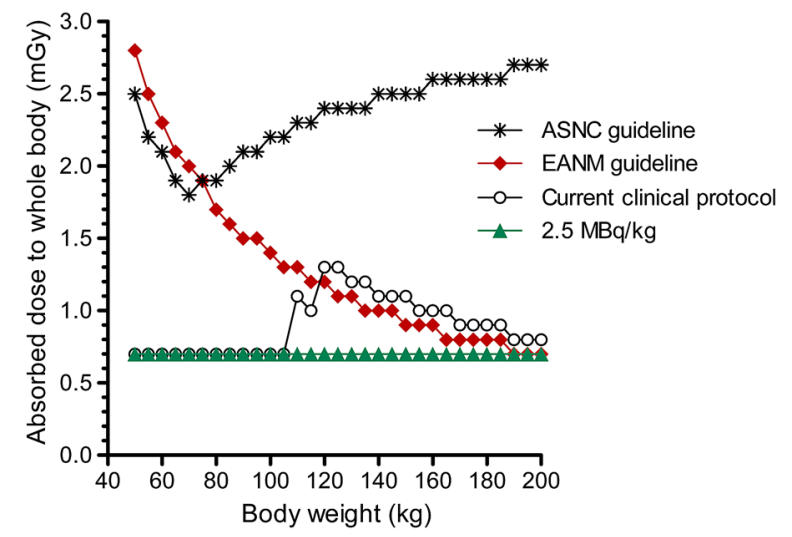

Figure 4. Mean absorbed dose from the stress examination to whole body as a function of body weight for administered activity at stress according to ASNC guidelines, EANM guidelines, our current clinical activity administration schedule, and $2.5 \mathrm{MBq} / \mathrm{kg}$.

\section{CONCLUSIONS}

A linear administration schedule of $2.5 \mathrm{MBq} / \mathrm{kg}$ body weight can be applied for MPS of patients within a wide range of body weight (51-193 kg, BMI 18-58) using either a CZT gamma camera or a conventional gamma camera with a resolution recovery reconstruction algorithm. Thus, the radiation dose associated with MPS can be significantly reduced in obese patients.

\section{Acknowledgments}

The authors would like to thank Christel Kullberg for excellent technical assistance. Financial support was provided by Swedish Heart-Lung Foundation, Lund University Faculty of Medicine, and Region of Scania.

\section{Disclosures}

The authors, J Oddstig, C Hindorf, $F$ Hedeer, J Jögi, $H$ Arheden, $M$ Hansson, and H Engblom, declare that they have no disclosures. 


\section{Open Access}

This article is distributed under the terms of the Creative Commons Attribution 4.0 International License (http:// creativecommons.org/licenses/by/4.0/), which permits unrestricted use, distribution, and reproduction in any medium, provided you give appropriate credit to the original author(s) and the source, provide a link to the Creative Commons license, and indicate if changes were made.

\section{References}

1. Lopez AD, Mathers CD, Ezzati M, Jamison DT, Murray CJ. Global and regional burden of disease and risk factors, 2001: Systematic analysis of population health data. Lancet. 2006;367:1747-57.

2. Gheorghiade M, Bonow RO. Chronic heart failure in the United States: A manifestation of coronary artery disease. Circulation. 1998;97:282-9.

3. Hachamovitch R, Berman DS, Shaw LJ, Kiat H, Cohen I, Cabico JA, Friedman J, Diamond GA. Incremental prognostic value of myocardial perfusion single photon emission computed tomography for the prediction of cardiac death: Differential stratification for risk of cardiac death and myocardial infarction. Circulation. 1998;97:535-43.

4. Hachamovitch R, Hayes S, Friedman J, Cohen I, Shaw L, Germano G. Determinants of risk and its temporal variation in patients with normal stress myocardial perfusion scans: What is the warranty period of a normal scan? J Am Coll Cardiol. 2003;41:132940.

5. Hachamovitch R, Rozanski A, Hayes SW, Thomson LE, Germano G, Friedman JD, Cohen I, Berman DS. Predicting therapeutic benefit from myocardial revascularization procedures: Are measurements of both resting left ventricular ejection fraction and stress-induced myocardial ischemia necessary? J Nucl Cardiol. 2006;13:768-78.

6. Authors/Task Force members, Windecker S, Kolh P, Alfonso F, Collet JP, Cremer J, Falk V, Filippatos G, Hamm C, Head SJ, Juni P, Kappetein AP, Kastrati A, Knuuti J, Landmesser U, Laufer G, Neumann FJ, Richter DJ, Schauerte P, Sousa Uva M, Stefanini GG, Taggart DP, Torracca L, Valgimigli M, Wijns W, Witkowski A. ESC/EACTS Guidelines on myocardial revascularization: The Task Force on myocardial revascularization of the European Society of Cardiology (ESC) and the European Association for Cardio-Thoracic Surgery (EACTS) developed with the special contribution of the European Association of Percutaneous Cardiovascular Interventions (EAPCI). Eur Heart J. 2014;2014:2541619.

7. Bocher M, Blevis IM, Tsukerman L, Shrem Y, Kovalski G, Volokh L. A fast cardiac gamma camera with dynamic SPECT capabilities: Design, system validation and future potential. Eur J Nucl Med Mol Imaging. 2010;37:1887-902.

8. Gambhir SS, Berman DS, Ziffer J, Nagler M, Sandler M, Patton J, Hutton B, Sharir T, Haim SB, Haim SB. A novel high-sensitivity rapid-acquisition single-photon cardiac imaging camera. J Nucl Med. 2009;50:635-43.

9. Hudson HM, Larkin RS. Accelerated image reconstruction using ordered subsets of projection data. IEEE Trans Med Imaging. 1994;13:601-9.

10. Modi BN, Brown JL, Kumar G, Driver RM, Kelion AD, Peters AM, Fowler JC. A qualitative and quantitative assessment of the impact of three processing algorithms with halving of study count statistics in myocardial perfusion imaging: Filtered backprojection, maximal likelihood expectation maximisation and ordered subset expectation maximisation with resolution recovery. J Nucl Cardiol. 2012;19:945-57.

11. Nkoulou R, Pazhenkottil AP, Kuest SM, Ghadri JR, Wolfrum M, Husmann L, Fiechter M, Buechel RR, Herzog BA, Koepfli P, Burger C, Gaemperli O, Kaufmann PA. Semiconductor detectors allow low-dose-low-dose 1-day SPECT myocardial perfusion imaging. J Nucl Med. 2011;52:1204-9.

12. Oddstig J, Hedeer F, Jogi J, Carlsson M, Hindorf C, Engblom H. Reduced administered activity, reduced acquisition time, and preserved image quality for the new CZT camera. J Nucl Cardiol. 2013;20:38-44.

13. International Commission on Radiological Protection. (ICRP) publication 105. Radiation protection in medicine. Ann ICRP. 2007;37:1-63.

14. Strauss HW, Miller DD, Wittry MD, Cerqueira MD, Garcia EV, Iskandrian AS, Schelbert HR, Wackers FJ, Balon HR, Lang O, Machac J. Procedure guideline for myocardial perfusion imaging 3.3. J Nucl Med Technol. 2008;36:155-61.

15. Verberne HJ, Acampa W, Anagnostopoulos C, Ballinger J, Bengel F, De Bondt P, Buechel RR, Cuocolo A, van Eck-Smit BL, Flotats A, Hacker M, Hindorf C, Kaufmann PA, Lindner O, Ljungberg M, Lonsdale M, Manrique A, Minarik D, Scholte AJ, Slart RH, Tragardh E, de Wit TC, Hesse B. EANM procedural guidelines for radionuclide myocardial perfusion imaging with SPECT and SPECT/CT: 2015 revision. Eur J Nucl Med Mol Imaging. 2015;42:1929-40.

16. Ali I, Ruddy TD, Almgrahi A, Anstett FG, Wells RG. Half-time SPECT myocardial perfusion imaging with attenuation correction. J Nucl Med. 2009;50:554-62.

17. Duvall WL, Sweeny JM, Croft LB, Ginsberg E, Guma KA, Henzlova MJ. Reduced stress dose with rapid acquisition CZT SPECT MPI in a non-obese clinical population: comparison to coronary angiography. J Nucl Cardiol. 2012;19:19-27.

18. Sharir T, Pinskiy M, Pardes A, Rochman A, Prokhorov V, Kovalski G, Merzon K, Bojko A, Brodkin B. Comparison of the diagnostic accuracies of very low stress-dose with standard-dose myocardial perfusion imaging: Automated quantification of oneday, stress-first SPECT using a CZT camera. J Nucl Cardiol. 2015;23:11-20.

19. van Dijk JD, Jager PL, Ottervanger JP, Slump CH, de Boer J, Oostdijk AH, van Dalen JA. Minimizing patient-specific tracer dose in myocardial perfusion imaging using CZT SPECT. J Nucl Med Technol. 2015;43:36-40.

20. Zafrir N, Solodky A, Ben-Shlomo A, Mats I, Nevzorov R, Battler A, Gutstein A. Feasibility of myocardial perfusion imaging with half the radiation dose using ordered-subset expectation maximization with resolution recovery software. J Nucl Cardiol. 2012;19:704-12.

21. Gimelli A, Bottai M, Giorgetti A, Genovesi D, Filidei E, Marzullo P. Evaluation of ischaemia in obese patients: Feasibility and accuracy of a low-dose protocol with a cadmium-zinc telluride camera. Eur J Nucl Med Mol Imaging. 2012;39:1254-61.

22. Herzog BA, Buechel RR, Katz R, Brueckner M, Husmann L, Burger IA, Pazhenkottil AP, Valenta I, Gaemperli O, Treyer V, Kaufmann PA. Nuclear myocardial perfusion imaging with a cadmium-zinc-telluride detector technique: Optimized protocol for scan time reduction. J Nucl Med. 2010;51:46-51.

23. Cerqueira MD, Weissman NJ, Dilsizian V, Jacobs AK, Kaul S, Laskey WK, Pennell DJ, Rumberger JA, Ryan T, Verani MS. Standardized myocardial segmentation and nomenclature for tomographic imaging of the heart: a statement for healthcare professionals from the Cardiac Imaging Committee of the Council 
on Clinical Cardiology of the American Heart Association. Circulation. 2002;105:539-42.

24. Fransson H, Ljungberg M, Carlsson M, Engblom H, Arheden H, Heiberg E. Validation of an automated method to quantify stressinduced ischemia and infarction in rest-stress myocardial perfusion SPECT. J Nucl Cardiol. 2014;21:503-18.

25. Holly TA, Abbott BG, Al-Mallah M, Calnon DA, Cohen MC, DiFilippo FP, Ficaro EP, Freeman MR, Hendel RC, Jain D, Leonard SM, Nichols KJ, Polk DM, Soman P. Single photonemission computed tomography. J Nucl Cardiol. 2010;17:941-73.

26. International Commission on Radiological Protection. (ICRP) publication 106. Radiation dose to patients from radiopharmaceuticals. Ann ICRP. 2008;38:1-197.
27. Fiechter M, Gebhard C, Fuchs TA, Ghadri JR, Stehli J, Kazakauskaite E, Herzog BA, Pazhenkottil AP, Gaemperli O, Kaufmann PA. Cadmium-zinc-telluride myocardial perfusion imaging in obese patients. J Nucl Med. 2012;53:1401-6.

28. Hindorf C, Oddstig J, Hedeer F, Hansson MJ, Jogi J, Engblom H. Importance of correct patient positioning in myocardial perfusion SPECT when using a CZT camera. J Nucl Cardiol. 2014;21:695702 .

29. Gimelli A, Bottai M, Quaranta A, Giorgetti A, Genovesi D, Marzullo P. Gender differences in the evaluation of coronary artery disease with a cadmium-zinc telluride camera. Eur J Nucl Med Mol Imaging. 2013;40:1542-8. 